\title{
Southern Fine Particulate Monitoring Project
}

\section{Fourth Quarterly Progress Report}

\author{
Reporting Period: July 1 - September 30, 2001
}

Issued: October 2001

DOE Cooperative Agreement No. DE-FC26-00NT40770

\author{
Submitted to \\ U.S. DEPARTMENT OF ENERGY \\ National Energy Technology Laboratory \\ P. O. Box 10940 \\ 626 Cochrans Mill Road \\ Pittsburgh, PA 15236-0940 \\ Attn: William Aljoe
}

\section{Prepared by}

SOUTHERN RESEARCH INSTITUTE

2000 Ninth Avenue South

P. O. Box 55305

Birmingham, AL 35255-5305

Principal Investigator: Ashley D. Williamson, (205) 581-2445 


\begin{abstract}
This quarterly report presents results and analysis of continuous onsite ambient fine particulate data at the North Birmingham study site during the July - September, 2000 study period. The continuous data include $\mathrm{PM}_{2.5}$ mass concentrations measured by TEOM, particle sulfate using the R\&P 8400S sulfate monitor, particle size distributions measured by SMPS and APS monitors, and $\mathrm{PM}_{2.5}$ light scattering extinction coefficient as measured by nephelometer. The report also presents some initial notes on our operating experience with the $8400 \mathrm{~S}$ sulfate analyzer. As described in the previous quarterly report, some persistent daily trends are seen in the particulate data, superimposed on a seasonal trend toward higher concentrations in warmer months. The sulfate mass fraction shows a markedly different time of day pattern from the balance of the particle mass, confirming the independent origin of this major mass fraction. The time variability of the major mass-bearing size fractions, and of the light-scattering potential, do not allow for a clean separation of independent size fractions. However, when the particle number averages are examined, the stronger time of day dependence of the smaller size fractions becomes more apparent, consistent with periods of higher formation of sub-100nm particles in early morning and in afternoon-evening periods.
\end{abstract}

\title{
Disclaimer
}

This report was prepared as an account of work sponsored by an agency of the United States Government. Neither the United States Government or any agency thereof, nor any of their employees, makes any warranty, express or implied, or assumes any legal liability or responsibility for the accuracy, completeness, or usefulness of any information, apparatus, product, or process disclosed, or represents that its use would not infringe privately owned rights. Reference herein to any specific commercial product, process, or service by trade name, trademark, manufacturer, or otherwise does not necessarily constitute or imply its endorsement, recommendation, or favoring by the United States Government or any agency thereof. The views and opinions of authors expressed herein do not necessarily state or reflect those of the United States Government or any agency thereof. 


\section{Table of Contents}

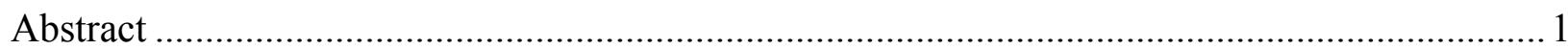

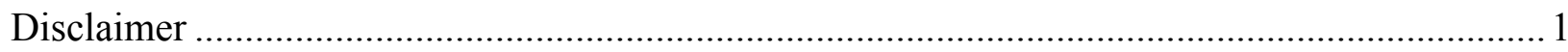

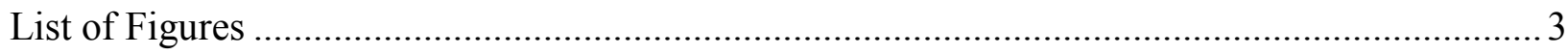

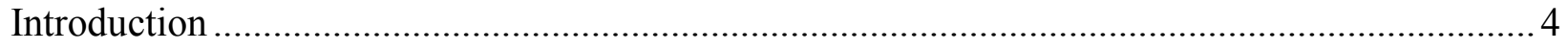

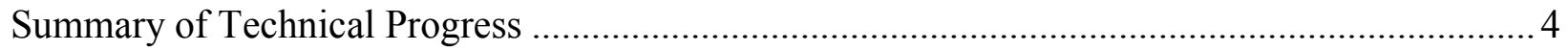

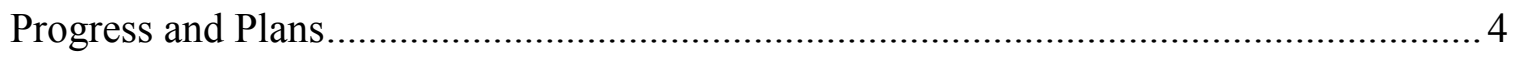

Problems and Assessment for Future Progress ................................................... 5

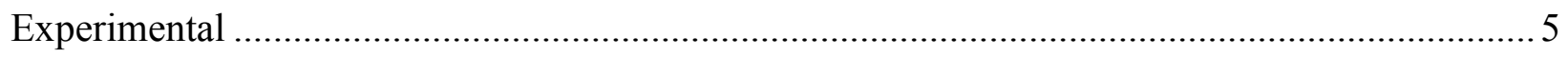

Assembly Of Instrumentation And Data Collection ............................................... 5

Initial Operating Experience With R\&P 8400S Sulfate Monitor................................... 5

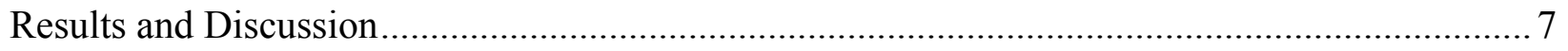

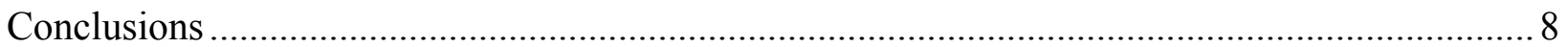




\section{List of Figures}

Figure 1. Hourly averaged fine particle data from the North Birmingham site during the period of July 1 - July $31,2001$.

Figure 2. Hourly averaged fine particle data from the North Birmingham site during the period of August 1 - August 31, 2001. 10

Figure 3. Hourly averaged fine particle data from the North Birmingham site during the period of September 1 - September 30, 2001.

Figure 4. Hourly averaged coarse particle data from the North Birmingham site during the period of July 1 - July 31, 2001. Also included are $\mathrm{PM}_{10}$ concentrations reported by Jefferson County

Figure 5. Hourly averaged coarse particle data from the North Birmingham site during the period of August 1 - August 31, 2001. Also included are $\mathrm{PM}_{10}$ concentrations reported by Jefferson County.

Figure 6. Hourly averaged coarse particle data from the North Birmingham site during the period of September 1 - September 30, 2001. Also included are $\mathrm{PM}_{10}$ concentrations reported by Jefferson County.

Figure 7. PM2.5 TEOM hourly time of day averages for each month of data collected at the North Birmingham site.

Figure 8. $\quad \mathrm{PM}_{2.5}$ TEOM, SMPS size fractions, and $8400 \mathrm{~S}$ sulfate hourly time of day averages for July 1 - July 31,2001 16

Figure 9. $\mathrm{PM}_{2.5}$ TEOM, SMPS size fractions, and $8400 \mathrm{~S}$ sulfate hourly time of day averages for August 1 - August 31, 2001.

Figure 10. $\mathrm{PM}_{2.5}$ TEOM, SMPS size fractions, and 8400 S sulfate hourly time of day averages for September 1 - September 30, 2001 18

Figure 11. Relative number concentration and nephelometer Bscat hourly time of day averages for July 1 - July 31, 2001.

Figure 12. Relative number concentration and nephelometer Bscat hourly time of day averages for August 1 - August 31, 2001.

Figure 13. Relative number concentration and nephelometer Bscat hourly time of day averages for September 1 - September 30, 2001.

Figure 14. Midnight to 10:00 hourly averaged SMPS particle size distributions for September 2001

Figure 15. Noon to 22:00 hourly averaged SMPS particle size distributions for September 2001. 


\section{Introduction}

This is the fourth quarterly progress report of the "Southern Fine Particulate Monitoring Project", funded by the U.S. Department of Energy's National Energy Technology Laboratory under DOE Cooperative Agreement No. DE-FC26-00NT40770 to Southern Research Institute (SRI). In this two year project SRI will conduct detailed studies of ambient fine particulate matter in the Birmingham, AL metropolitan area. Project objectives include:

Augment existing measurements of primary and secondary aerosols at an established urban southeastern monitoring site

Make a detailed database of near-continuous measurements of the time variation of fine particulate mass, composition, and key properties (including particle size distribution) Apply the measurements to source attribution, time/transport properties of fine PM, and implications for management strategies for $\mathrm{PM}_{2.5}$

Validate and compare key measurement methods used in this study for applicability within other $\mathrm{PM}_{2.5}$ research by DOE-FE, EPA, NARSTO, and others.

\section{Summary of Technical Progress}

\section{$\underline{\text { Progress and Plans }}$}

During the fourth project quarter, continuous onsite ambient data were collected and monitored. Details include:

- July measurement intensive with Eastern Supersite program

- $\quad$ Continued laboratory testing for particulate sulfate monitor of Harvard design

- Installation of the 43CTL Sulfur Dioxide Analyzer

- Continued monitoring with TEOM, particle sizing instruments, R\&P 8400 Sulfate monitor and Nephelometer

- $\quad$ Paper presented at American Chemical Society meeting in Chicago, IL.

Plans for next quarter include the following:

Field study comparisons for ambient and dried measurements using TEOM, Nephelometer, and particle size package Installation of particulate sulfate monitor of Harvard design; onsite comparative measurements with R\&P 8400S and SEARCH Particulate Composition Matter (PCM) results Verify 8400 S data and compare with PCM sulfate concentrations Prepare July data in general format for modeling study Upgrade the TSI APS 3320 to an APS 3321

Continue onsite monitoring with continuous monitoring instruments Continue analysis of initial continuous particulate data 


\section{$\underline{\text { Problems and Assessment for Future Progress }}$}

Minor computer difficulties in early July caused some data loss for the particle sizing package. The data losses were not significant to our collection of data. Some initial operating problems were experienced with the Rupprecht \& Pataschnick 8400S sulfate analyzer. Outright data losses have been minimal, but instrument drift has led to possible biases in the data taken during the July ESP01 period. Further tests are in progress to assess and minimize the resulting data uncertainties.

\section{Experimental}

\section{$\underline{\text { Assembly Of Instrumentation And Data Collection }}$}

After the installation of the new shelter to the North Birmingham air monitoring station in June, the configuration for APS and SMPS particle sizing instruments was updated. A standard PM 10 inlet was installed to increase the counting efficiency in the larger size ranges of the particle size package. A 1-Liter time averaging chamber was installed directly before the SMPS inlet. The chamber was installed to average short term fluctuations in ambient concentrations over the five minute scan cycle.

During the period 6/30/2001 - 8/3/2001, our project participated with other measurement groups in the Eastern Supersites Program summer intensive program (ESP01). During this period we collected continuous monitoring data from all instruments in the North Birmingham shelter. All data have been compiled and are undergoing QA screening. After Level 1 data are complete, the data set will be forwarded to the EPA program contact for inclusion in the ESP01 modeling study.

\section{Initial Operating Experience With R\&P 8400S Sulfate Monitor}

The Rupprecht \& Pataschnick 8400S sulfate analyzer was deployed at the North Birmingham site on June 19. Ambient sulfate measurements using the monitor were collected throughout the quarter with minor interruptions. The data will be discussed in more detail below; in this section we present some initial notes on our operating experience with the instrument.

Generally, the instrument operates well in unattended mode for long periods, with minimal operator interaction required and with minimal data loss due to instrument status faults. However, several operating problems have occurred during the quarter, some specific to our instrument and others which are probably more generally applicable with the design of the $8400 \mathrm{~S}$. The most significant problem has been excessive drift in the instrument sensitivity, as monitored by daily span gas audits. After calibration of the $\mathrm{SO}_{2}$ pulse analyzer when the $8400 \mathrm{~S}$ was deployed, we observed a fairly steady drop in steady state audit concentration readings, reaching approximately $8 \%$ below the certified tank value after a month of operation. On $7 / 21$ the analyzer was recalibrated, after which the audit values again dropped until on $8 / 6$ the analyzer response was again $8-9 \%$ low and was again recalibrated. During the next week the loss of sensitivity accelerated, and we performed further diagnostic tests on 8/14. At this time we discovered that the temperature/ pressure correction algorithm on the $\mathrm{SO}_{2}$ pulse analyzer had 
been disabled before the instrument was delivered, and that the UV lamp intensity had decreased to about $30 \%$ of its original value. Due to operating changes, such as loading of the analyzer prefilter, the temperature/ pressure correction would be expected to cause a reduction in instrument response as the instrument pressure drop increases. The actual decrease in response was several times what could reasonably be expected from this effect. The effects of the lamp intensity loss were less clear, since the analyzer circuitry provides a normalized response to compensate for lamp changes. Further tests have suggested that this compensation was inadequate.

On August 14-15, a "Factory Cal" was performed on the analyzer: the lamp position was adjusted to "peak" the intensity on the UV detector, and the amplifier gains were readjusted. The temperature/ pressure correction was enabled at this time. After peaking, the lamp intensity signal was roughly $2500 \mathrm{mV}$, about 60 percent of the original value. The lamp intensity value has been recorded manually since these adjustments and was observed to vary somewhat erratically between 1700 and $3500 \mathrm{mV}$ over the ensuing period. We found that while the instrument's internal correction factor does linearly track the lamp intensity, the apparent concentration of the audit gas varied linearly from $+2.5 \%$ to $-3 \%$ as the voltage decreased from 3500 to $2000 \mathrm{mV}$, and appears to drop off more than linearly below $2000 \mathrm{mV}$. We plan to develop a correction factor based on the audit data after some further experiments. We anticipate that the systematic error due to lamp variability will be less than 10 percent, which should be reducible to 1-3 percent by applying the compensation factors to the existing data. After consultation with R\&P, the vendor agreed to send a replacement lamp that will be installed in the hope of greater stability in the future.

A second problem was noted as soon as outdoor sampling was started. The sample flowrate, initially above $1.1 \mathrm{l} / \mathrm{min}$, steadily dropped as the sample orifice presumably became occluded due to upstream impaction of the humidified droplets. The rate of decline was greatest when the outside dewpoint was high, but the flow generally drops near the $0.8 \mathrm{l} / \mathrm{min}$ alarm limit in $7-10$ days, requiring the orifice to be removed and cleaned. In principle the instrument operates well over the range of sample flow encountered, but the maintenance frequency is higher than desirable.

As reported last quarter, we assembled a prototype sulfate monitor on the design of George Allen from the Harvard School of Public Health (HSPH) and made initial tests in the laboratory. Initial testing concentrated on optimizing the reactor length and materials and on determining the relative response of different reactor configurations and operating temperatures. Following the ARA design changes, stainless steel tubing heated in a tube furnace was used for all reactor configurations. Parallel testing of dual tube/furnace configurations allowed independent variation of tube geometry and temperature effects. Further tests of the reactor tube configuration and of preliminary denuders were performed during July, and orders for other reactor tube materials were placed for additional tests. In the interim the Thermo 43CTL SO2 monitor was installed at the North Birmingham site in August, for comparison with the SO2 analyzer of the R\&P 8400S. Results of these studies will be presented in a future report. 


\section{Results and Discussion}

Hourly averages of the continuous particulate measurements are presented in Figures $1-6$. The data are plotted together for a meaningful comparison between instruments and data sets. The figures contain the $\mathrm{PM}_{2.5}$ mass concentrations measured by the TEOM, 8400S sulfate monitor and integrated size fractions measured by the particle sizing devices. Included are total (submicron) mass concentration as derived from the SMPS measurements, and integrated mass concentrations in the $1-2.5$ and $2.5-10 \mu \mathrm{m}$ size ranges from the APS measurement data. The $\mathrm{PM}_{2.5}$ light scattering extinction coefficient as measured by the M903 nephelometer is plotted on the second $\mathrm{Y}$-axis. In addition, hourly average $\mathrm{PM}_{10}$ concentration data were obtained from the Jefferson County Health Department as measured by the county TEOM monitor at the site.

Figures $1-3$ display the variables associated with the measurements in the fine particulate region. The data sets displayed are the $\mathrm{PM}_{2.5}$ TEOM, SMPS total concentration, the $1-2.5 \mu \mathrm{m}$ APS fraction, the $8400 \mathrm{~S}$ sulfate monitor and the M903 nephelometer. The figures present the same five variables over the months July, August, and September, respectively. Figures 4 - 6 represent the variables associated with particulate measurements in the coarse size region, including the $\mathrm{PM}_{10}$ TEOM and the $2.5-10 \mu \mathrm{m}$ APS fraction, as well as the $\mathrm{PM}_{2.5}$ TEOM concentrations for reference.

As described in the previous quarterly report, some persistent daily trends are seen in the particulate data, superimposed on a seasonal trend toward higher concentrations in warmer months. To depict $\mathrm{PM}_{2.5}$ trends during the day, the $\mathrm{PM}_{2.5}$ TEOM mass concentration hourly averages were averaged with the corresponding hour for each day throughout the month. Figure 7 displays this comparison for each month our data have been collected at the North Birmingham site. Overnight steady high levels followed by rush hour peaks are seen to some extent during each month of data collection. After the morning peak, average concentrations dropped to a lower level during daylight hours, followed by a steady rise from late afternoon to midnight. The steady daytime concentrations are in the $16-19 \mu \mathrm{g} / \mathrm{m}^{3}$ range during the spring months and September, but trend to higher levels in the summer months with suggestions of a mid afternoon increase. The overnight concentrations and morning rush hour peak are more variable from month to month, even within seasons. Generally, concentrations in these hours are lower in the spring and fall months when the air is typically cleaner.

Figures $8-10$ display hourly average concentrations of several components of the fine mass concentration for July through September. As in Figure 7, the fine particle size ranges and the sulfate fraction hourly averages were averaged with the corresponding hour for all days within each month. In each figure, mass concentrations of total sulfate and particulate mass in five submicron size ranges are plotted. The $\mathrm{PM}_{2.5}$ TEOM mass concentration is scaled for visibility and plotted on the second $\mathrm{Y}$-axis as a reference. All size fractions qualitatively follow the same trend including the broad afternoon increase in July and August. The major mass-bearing size fractions $(120-900 \mathrm{~nm})$ show the same overall trends as the $\mathrm{PM}_{2.5}$ TEOM mass concentration, with the morning rush hour maximum slightly less pronounced. In contrast, the sulfate concentration shows very little sign of a morning rush hour peak, but for all three months does show a broad maximum in the daytime hours. 
Although all size fractions do show the same qualitative hourly trends described above, there are size-specific quantitative differences in behavior. To illustrate this, Figures $11-13$ depict the relative behavior of the fine aerosol fractions throughout the day. Each hourly averaged concentration (on a number basis) is normalized to the 24 hour concentration average of the respective size range. On the number basis, the overnight concentration increase is progressively more pronounced for the smaller size fractions. The relative number concentration for the two largest size fractions shows comparatively little change throughout the day. Plotted on the same basis, the nephelometer Bscat readings follow the same trend as the larger two submicron fractions, as might be expected from a surface area distribution.

To provide a more detailed view of the evolution of the particle size distribution during the day, Figures 14 and 15 detail the hourly averaged SMPS particle size distributions for September. The particle size distributions are based on hourly average number concentrations throughout the day. Figure 14 contains, at 2 hour intervals, the hourly average size distributions from midnight to 10:00, with the maximum number concentration at $4: 00\left(5.8 \mathrm{E} 8 \# / \mathrm{m}^{3}\right.$ at $\left.62.6 \mathrm{~nm}\right)$. The stable size distribution at midnight and 2:00 is augmented by the ingrowth of new fine particles in the 4:00- 6:00 period, after which the number of particles decreases and the size distribution broadens through the rest of the morning hours, suggesting that the particle mass ages as it is diluted by mixing. Figure 15 displays the size distributions from noon to 22:00. It appears that the aged morning aerosol is augmented by a new mode of fine particles in early afternoon, followed by a steady ingrowth of a mode centered at $60 \mathrm{~nm}$ from 18:00 through the rest of the day.

\section{Conclusions}

The time of day analyses shown in Figures 7-15 indicate some significant trends regarding the sources and evolution of the fine particle concentrations in this urban area. The time of day pattern seen in Figure 7 for $\mathrm{PM}_{2.5}$ mass is qualitatively similar through the months of the study. The fact that the sulfate mass fraction shows a markedly different time of day pattern in Figures 8-10 confirms the independent origin of this major mass fraction from the balance of the particle mass. The time variability of the major mass-bearing size fractions and of the light-scattering potential do not allow for as clean a separation of independent size fractions. However, when the particle number averages are examined in Figures 11-13, the stronger time of day dependence of the smaller size fractions becomes more apparent, consistent with periods of higher formation of sub-100nm particles in early morning and in afternoon-evening periods. More detailed time dependence analyses should be useful in separating the effects of different local and distant sources. 


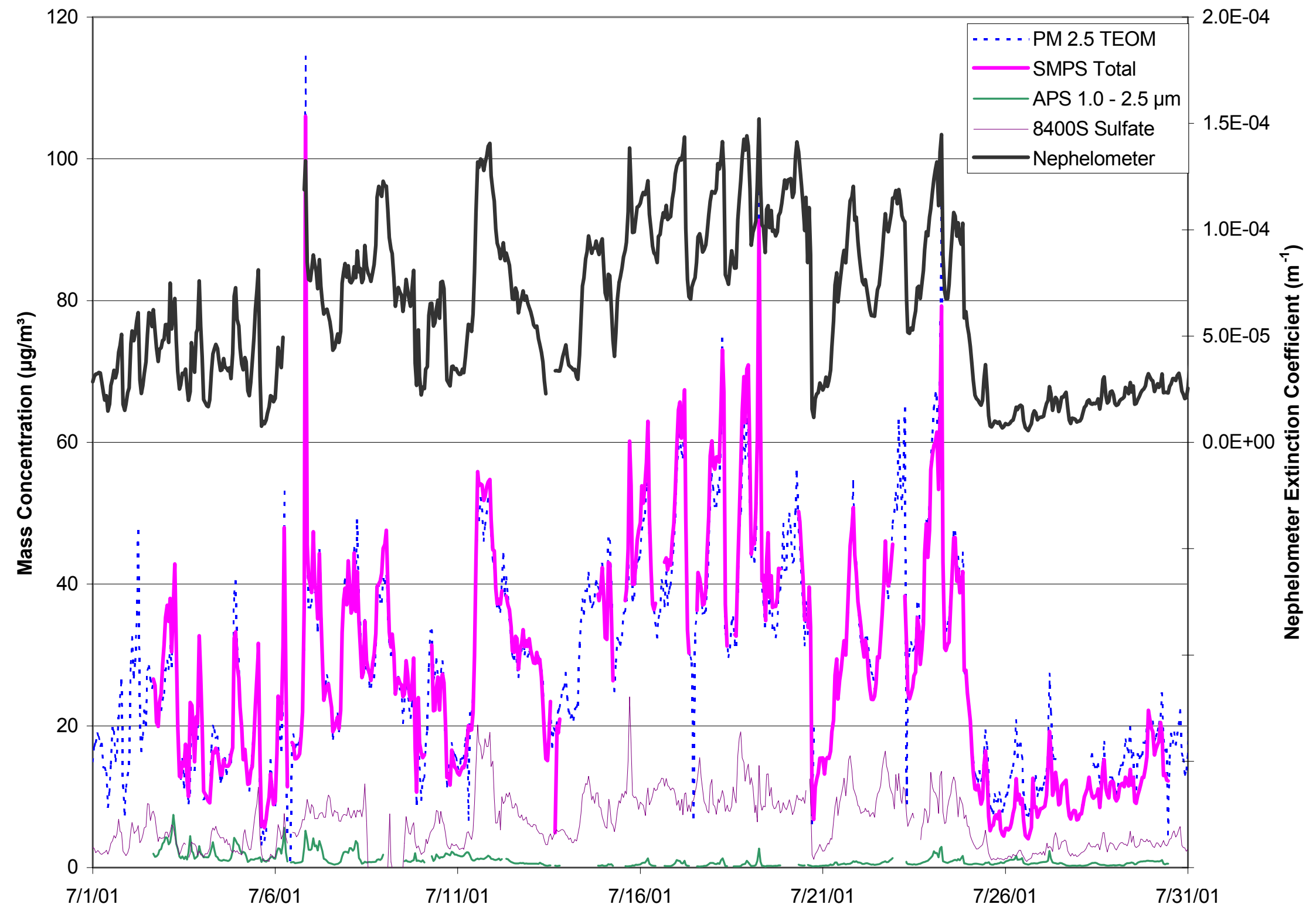

Figure 1. Hourly averaged fine particle data from the North Birmingham site during the period of July $1-$ July 31,2001 . 


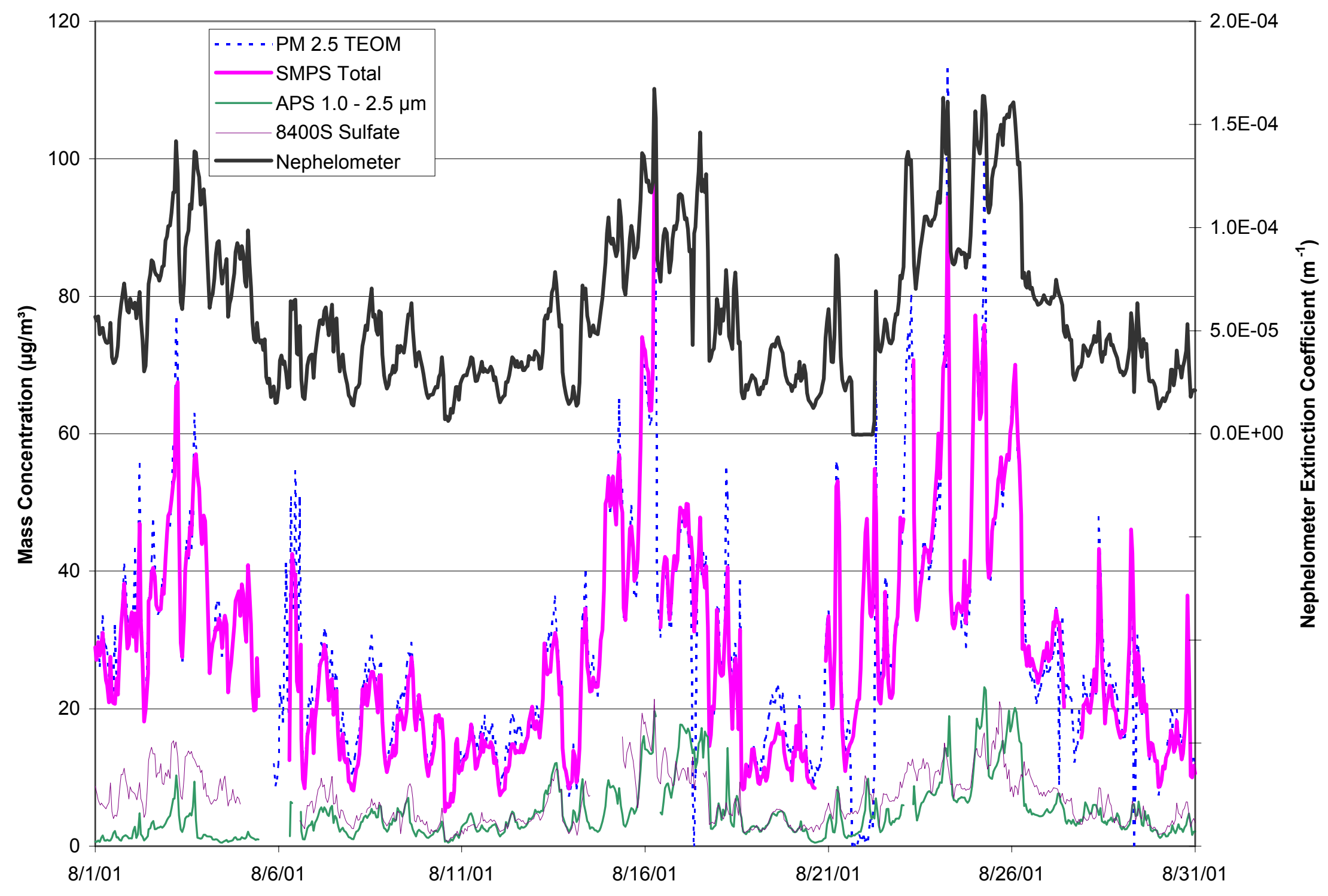

Figure 2. Hourly averaged fine particle data from the North Birmingham site during the period of August 1 - August 31, 2001. 


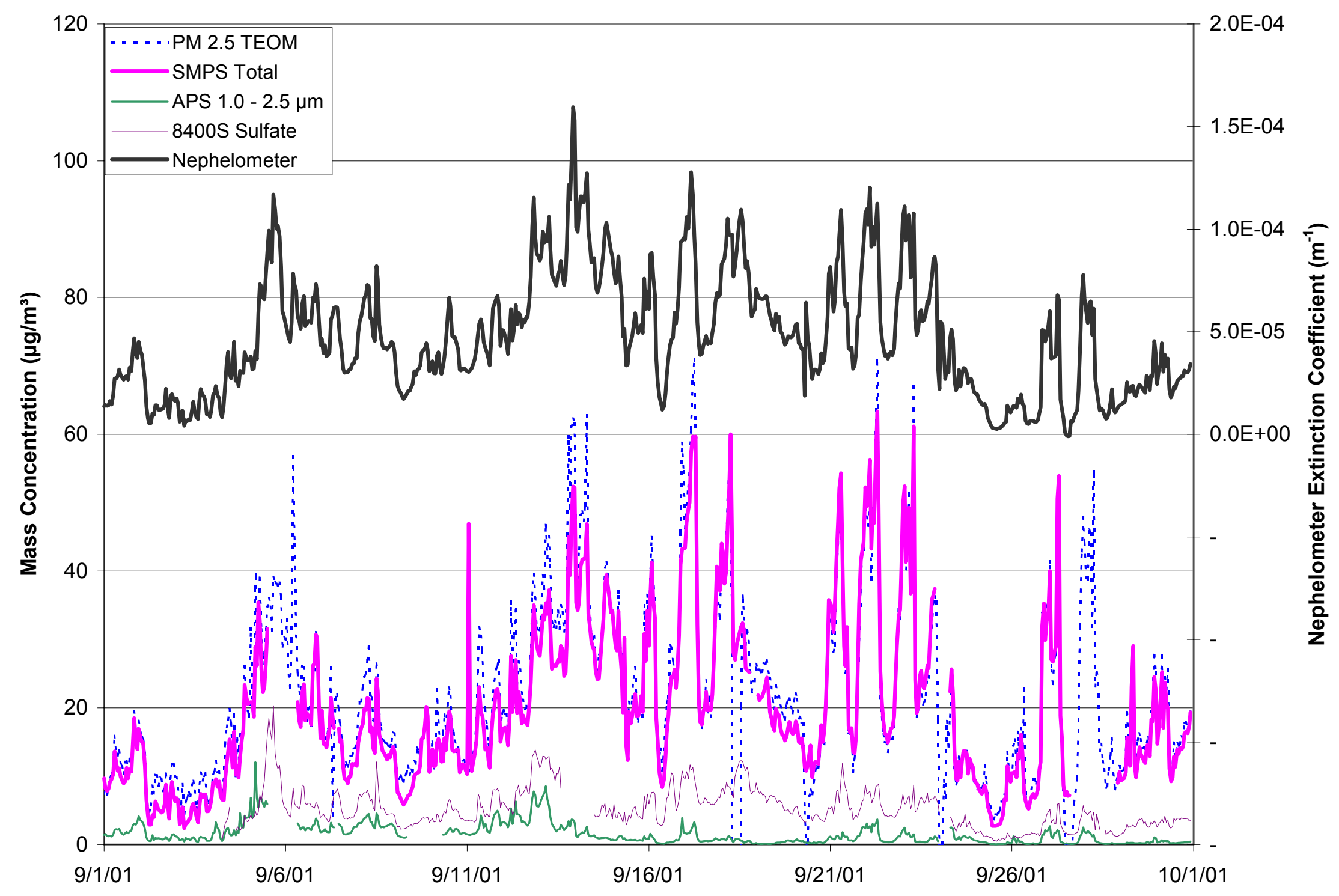

Figure 3. Hourly averaged fine particle data from the North Birmingham site during the period of September 1 - September 30, 2001. 


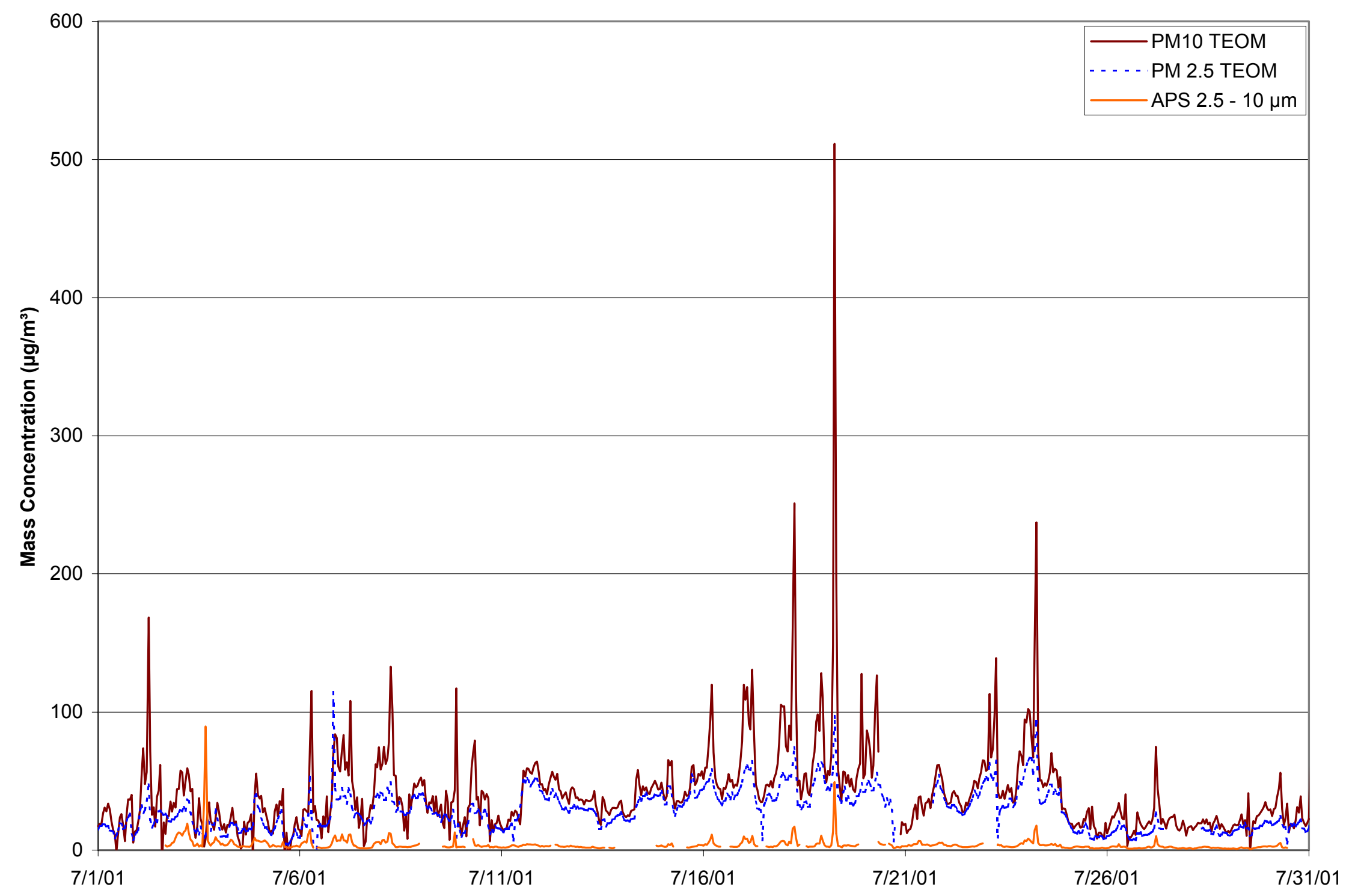

Figure 4. Hourly averaged coarse particle data from the North Birmingham site during the period of July 1 - July $31,2001$. Also included are $\mathbf{P M}_{10}$ concentrations reported by Jefferson County 


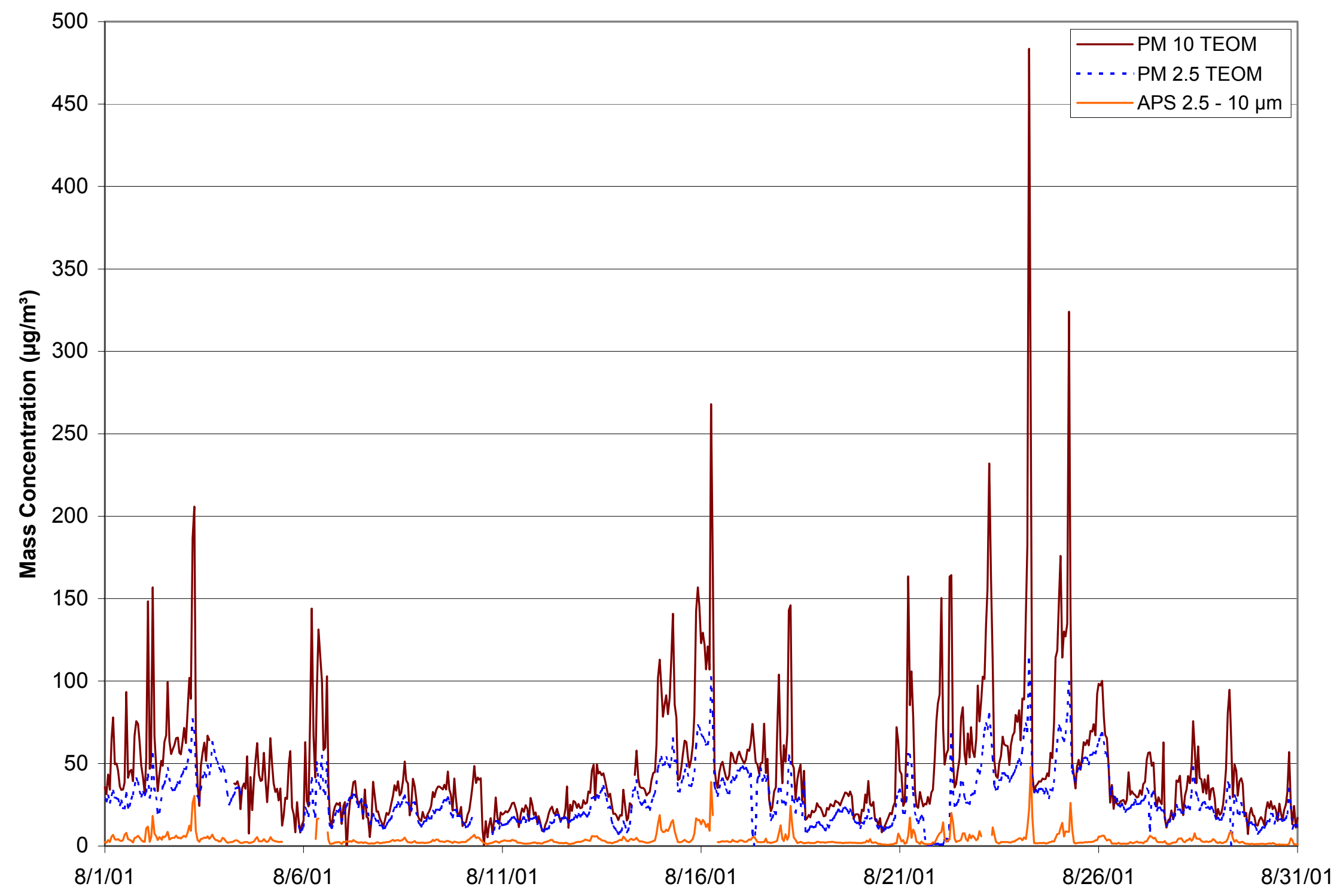

Figure 5. Hourly averaged coarse particle data from the North Birmingham site during the period of August 1 - August 31, 2001. Also included are $\mathbf{P M}_{10}$ concentrations reported by Jefferson County. 


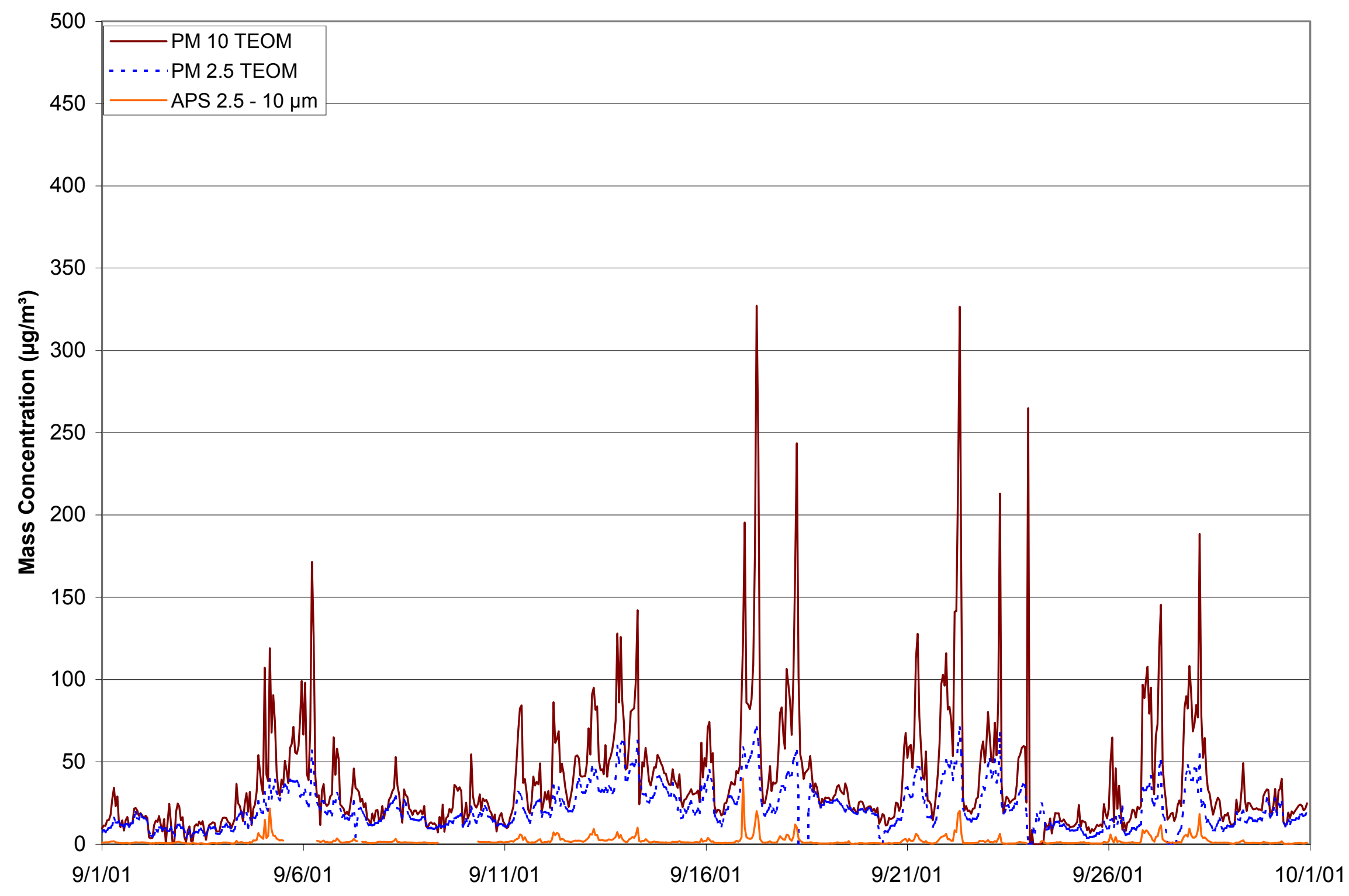

Figure 6. Hourly averaged coarse particle data from the North Birmingham site during the period of September 1 - September 30, 2001. Also included are $\mathbf{P M}_{10}$ concentrations reported by Jefferson County. 


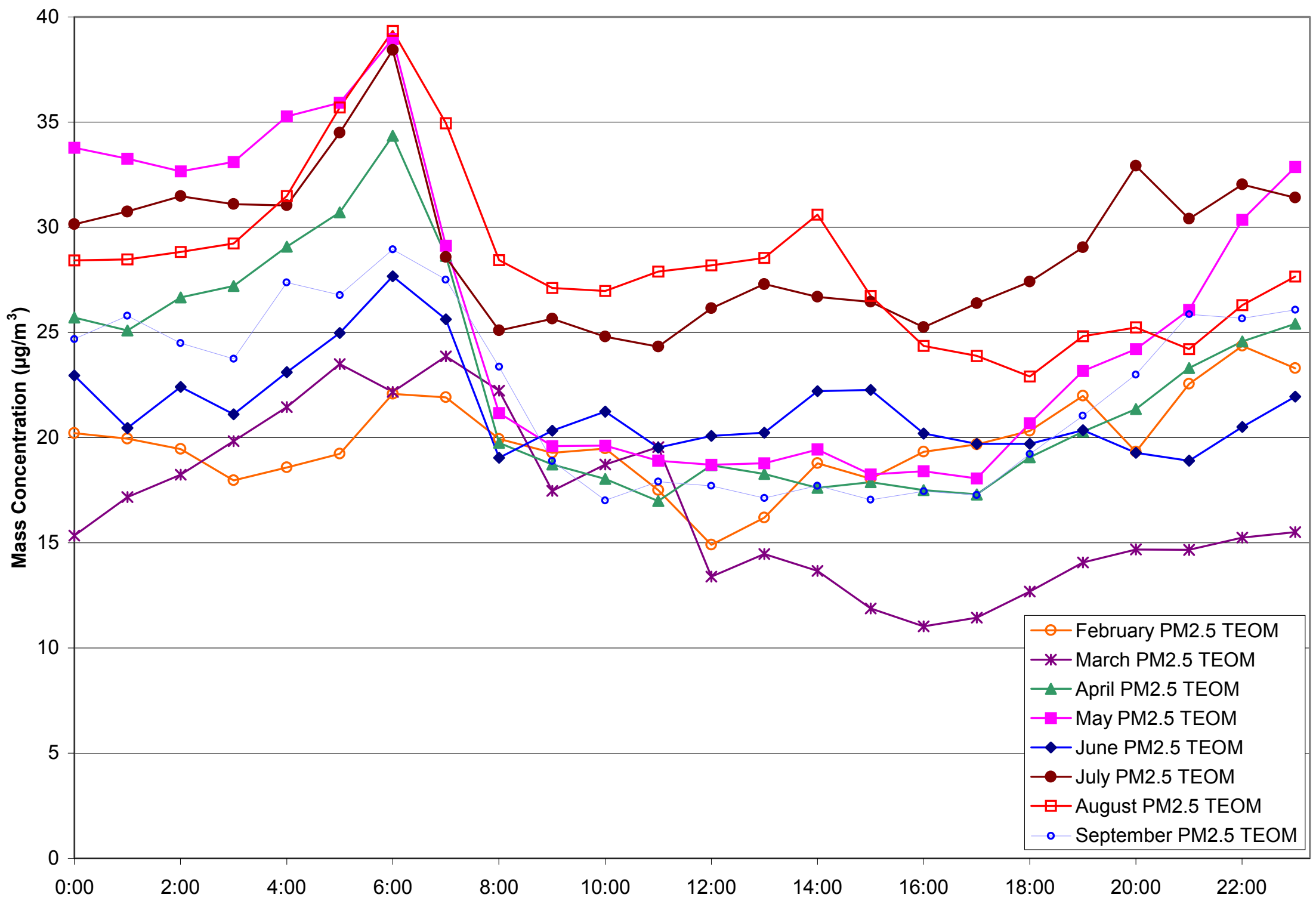

Figure 7. PM2.5 TEOM hourly time of day averages for each month of data collected at the North Birmingham site. 


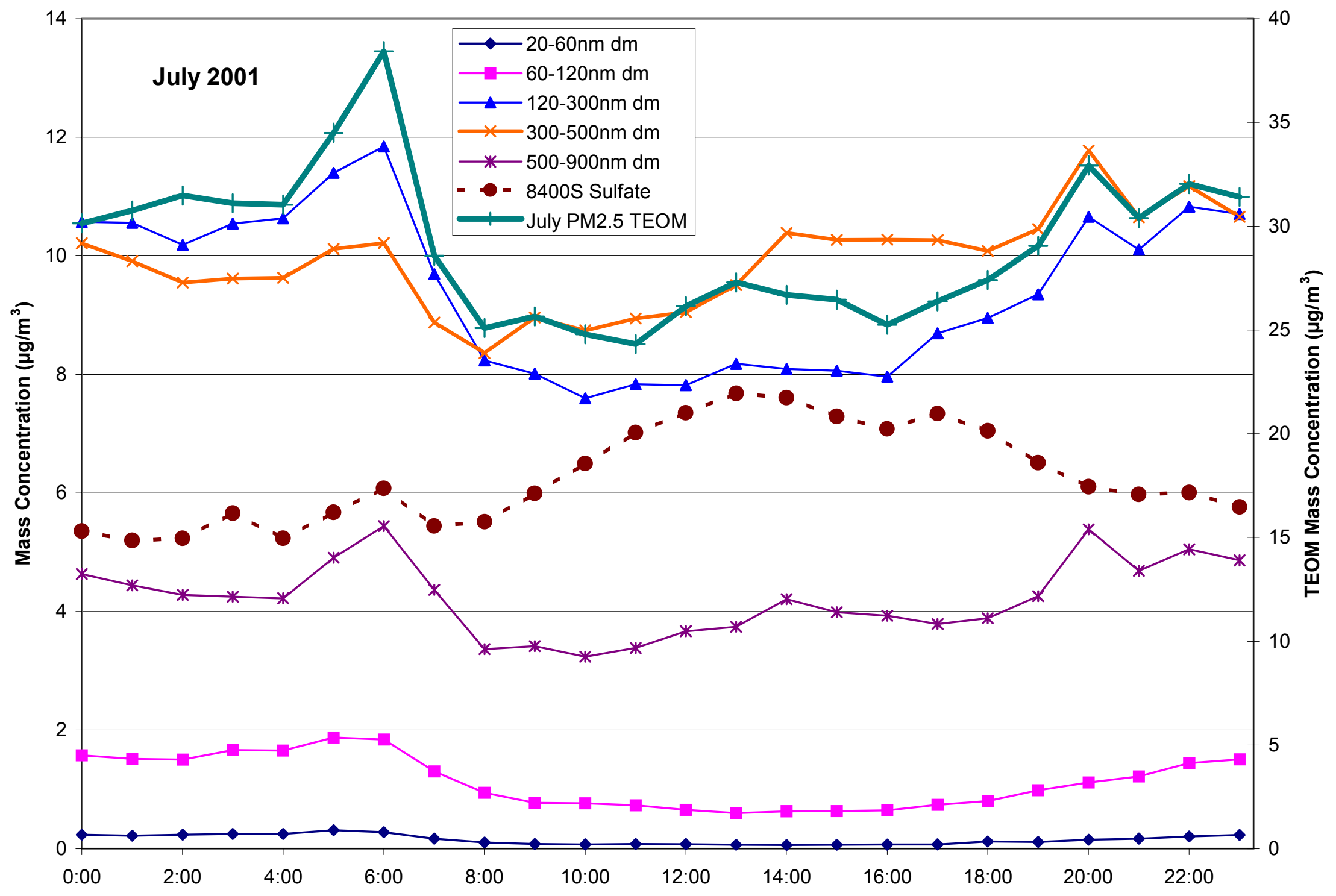

Figure 8. $\quad$ PM $_{2.5}$ TEOM, SMPS size fractions, and 8400S sulfate hourly time of day averages for July 1 - July 31, 2001. 


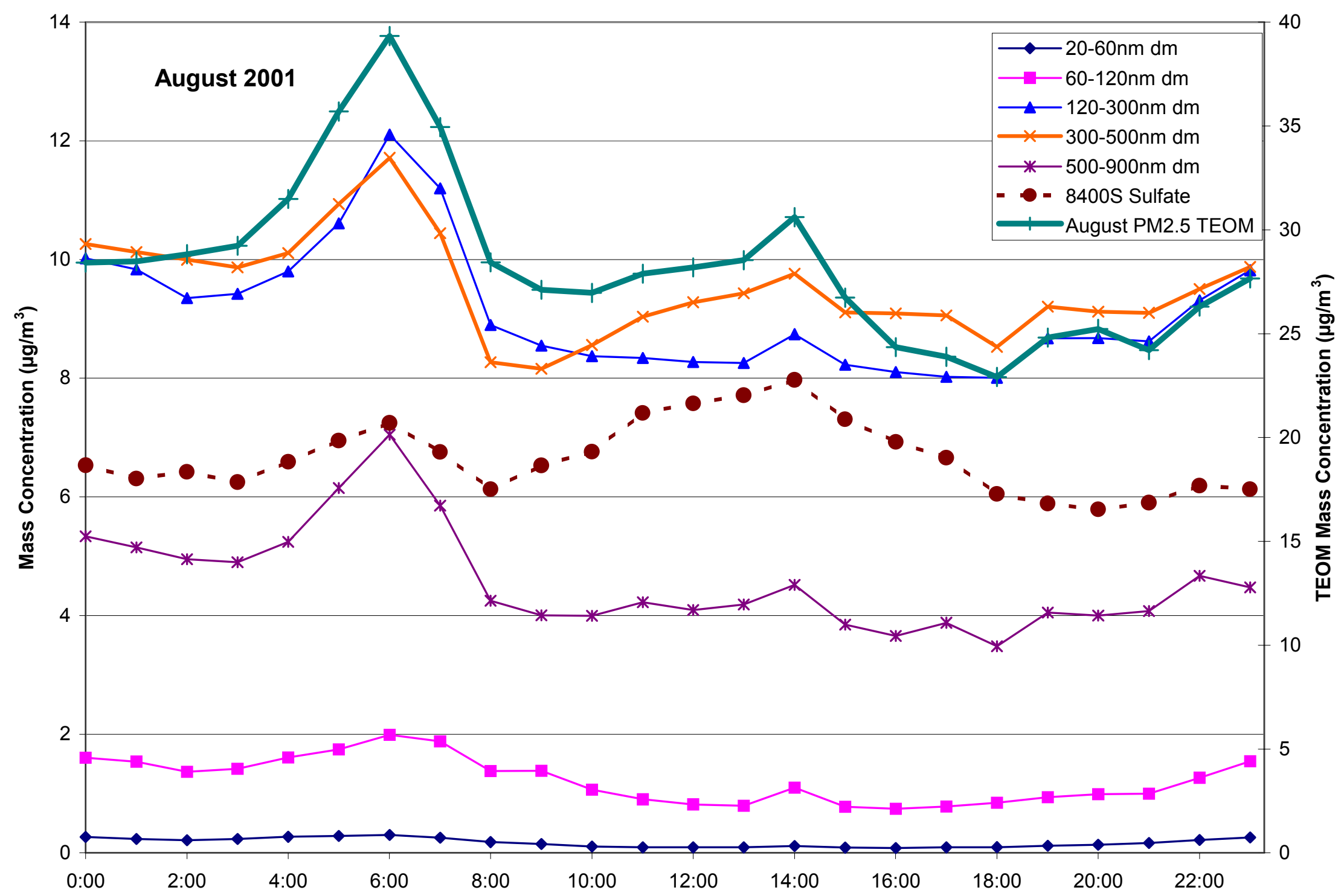

Figure 9. PM $\quad$ PM.5 $_{.5}$ TEOM, SMPS size fractions, and 8400S sulfate hourly time of day averages for August 1 - August 31, 2001. 


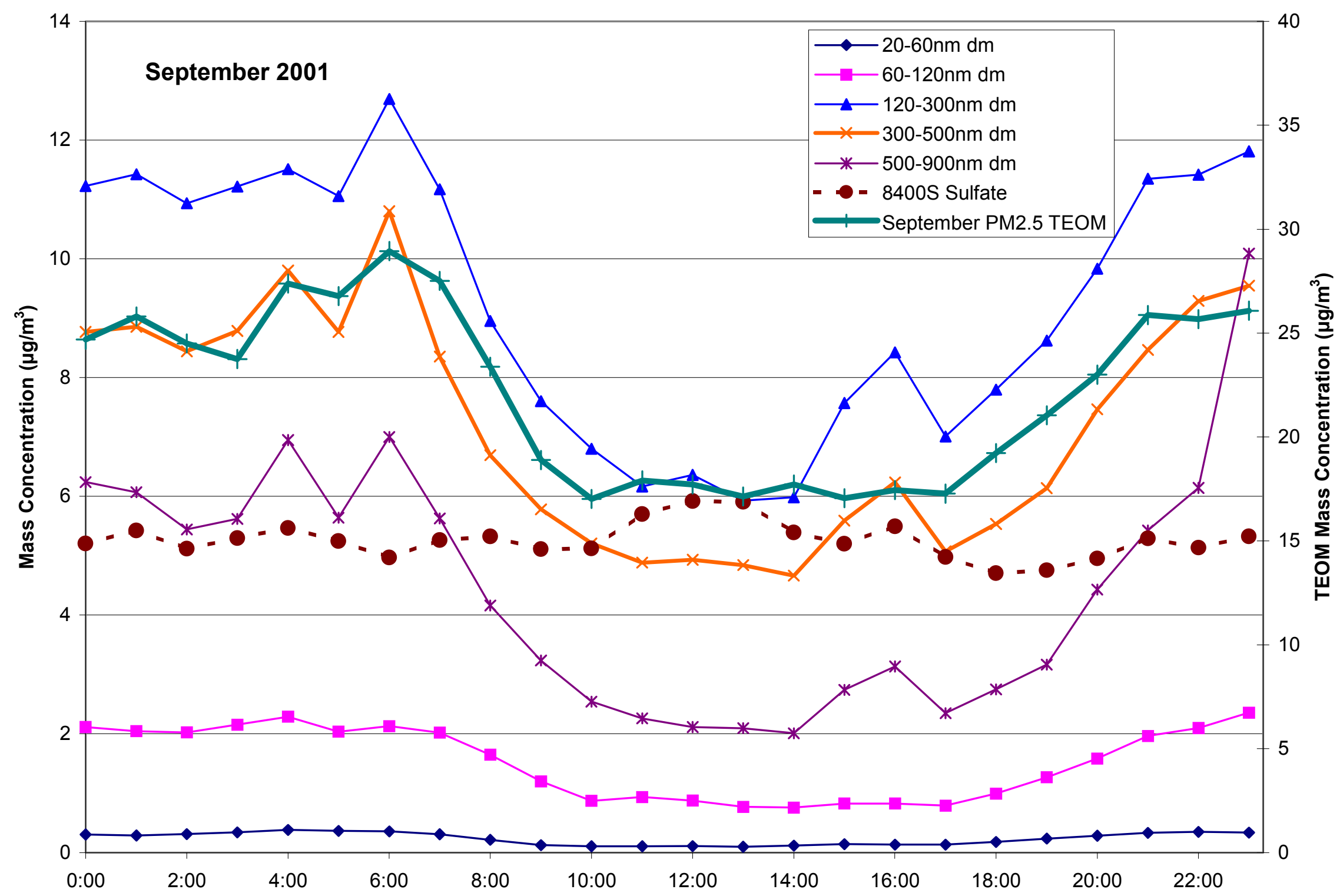

Figure 10. PM $_{2.5}$ TEOM, SMPS size fractions, and 8400 S sulfate hourly time of day averages for September 1 - September 30 , 2001. 


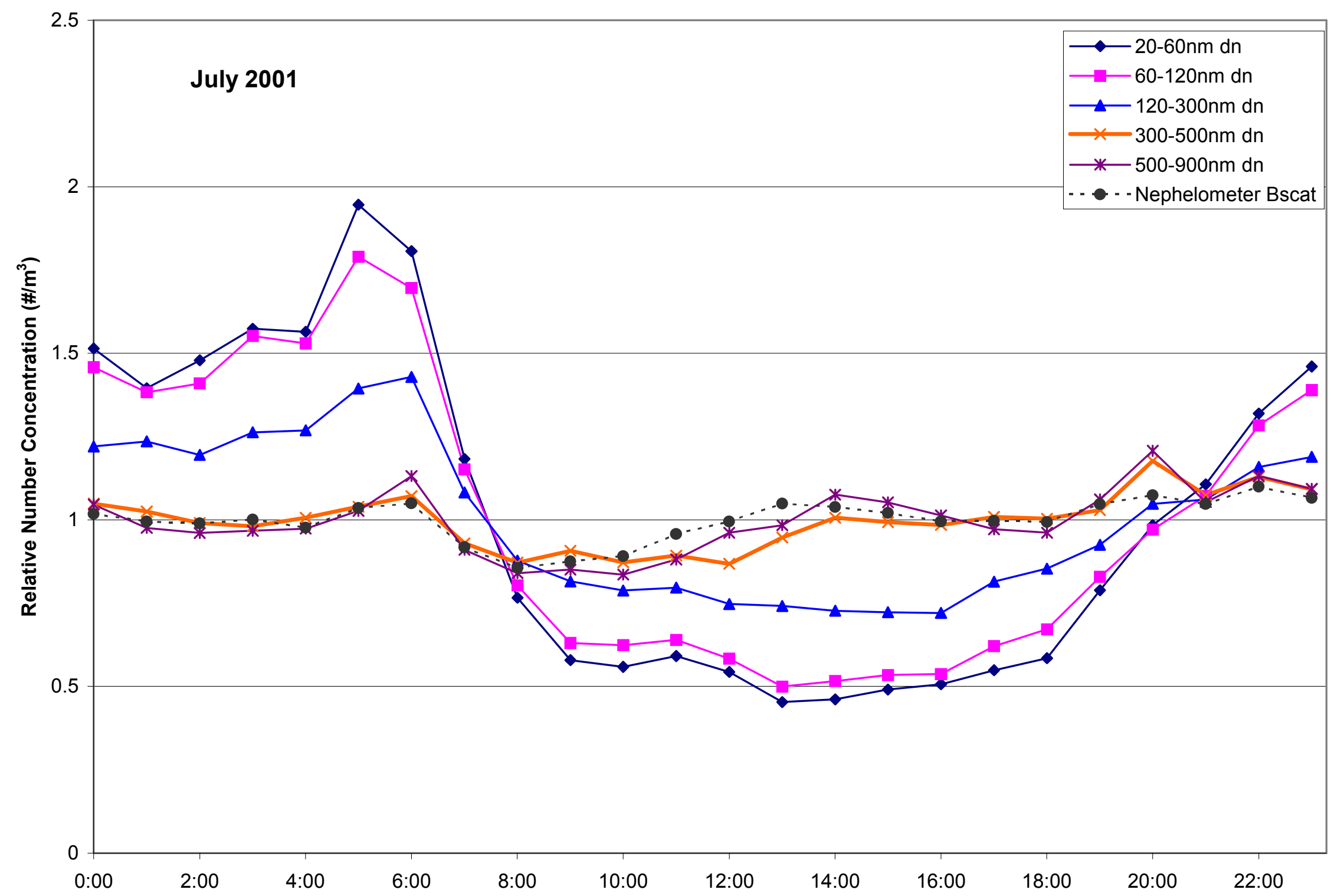

Figure 11. Relative number concentration and nephelometer Bscat hourly time of day averages for July 1 - July $31,2001$. 


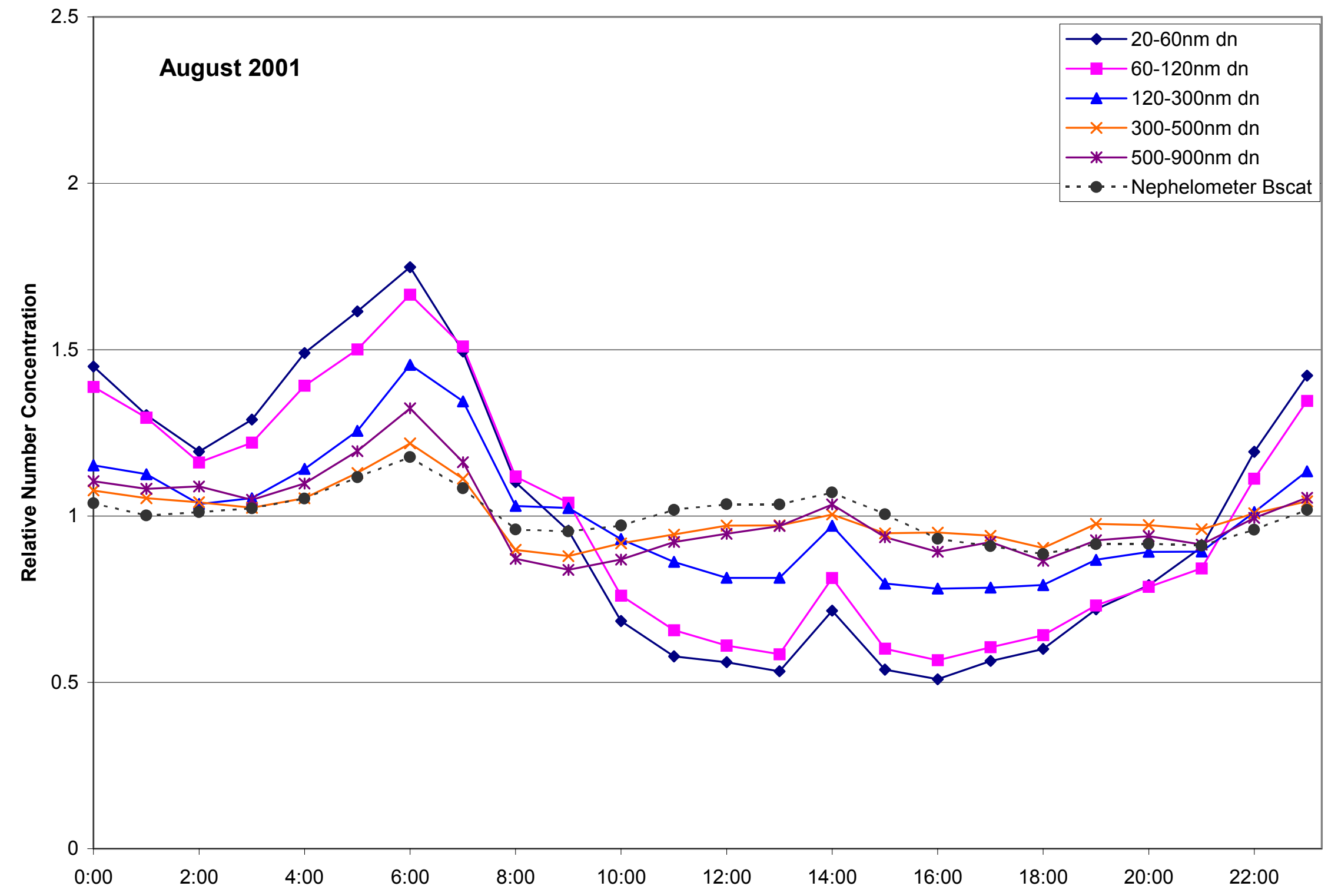

Figure 12. Relative number concentration and nephelometer Bscat hourly time of day averages for August 1 - August 31, 2001. 


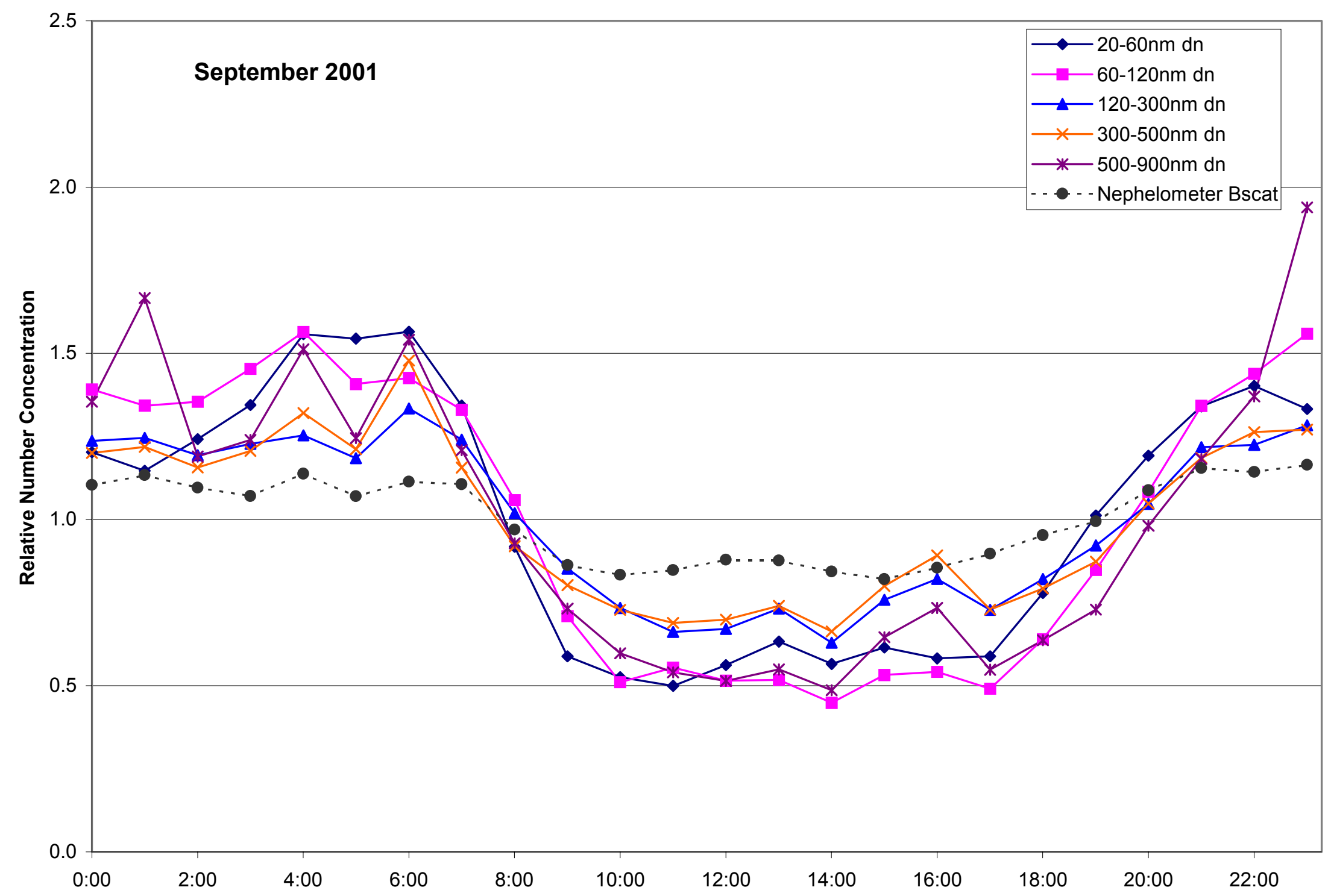

Figure 13. Relative number concentration and nephelometer Bscat hourly time of day averages for September 1 - September 30, 2001. 


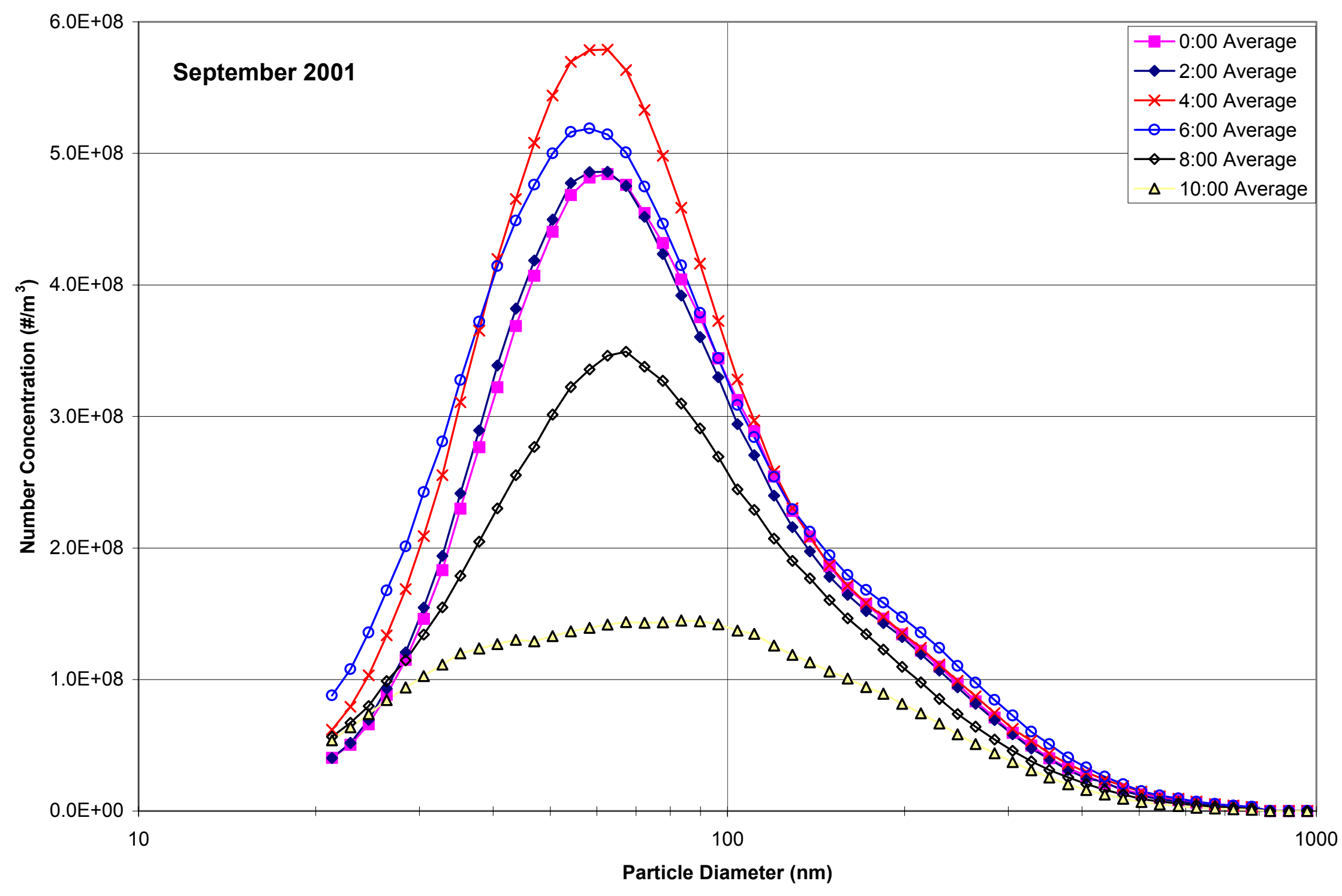

Figure 14. Midnight to 10:00 hourly averaged SMPS particle size distributions for September 2001. 


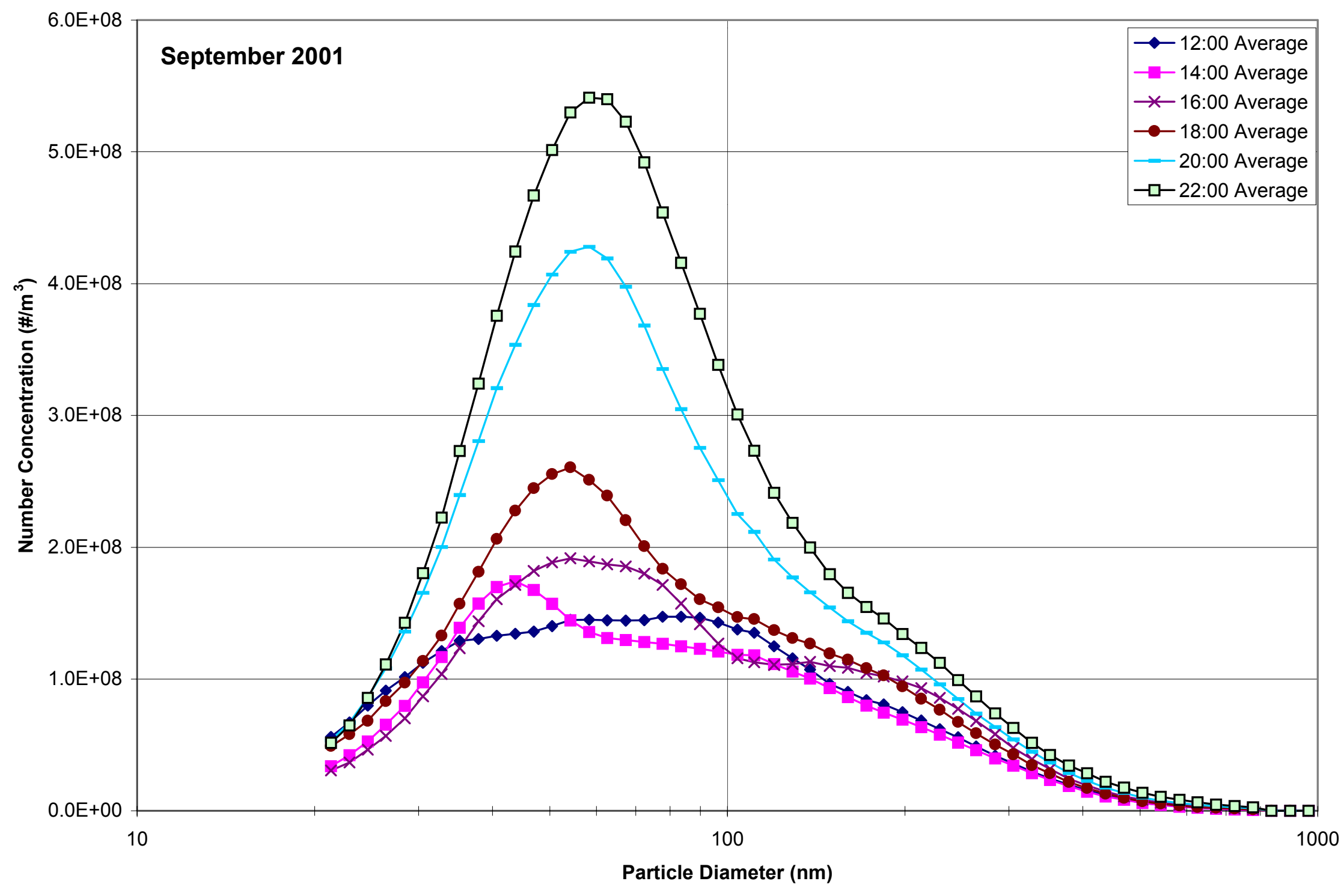

Figure 15. Noon to 22:00 hourly averaged SMPS particle size distributions for September 2001. 